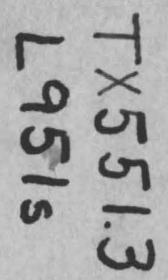

\title{
Score Your Diet
}

prepared by

MIRIAM E. LOWENBerG, Ph.D.

College of Home Economics

Pennsylvania State University

University Park, Pennsylvania

MILDRED MOUW, R.N., M.A.

Department of Health

Pittsburgh,

Pennsylvania
LAURA WEYRICK HOEFT, R.N., M.A.

Crippled Children's Service

Department of Public Welfare

Bemidji, Minnesota

DOLORES NYHUS, B.A., M.S.

State of California

Department of Public Health

San Francisco, California 


\section{$T \times 551.3$}

\section{L95 Is}

\section{HOW TO SCORE YOUR DIET}

This score sheet is a device by which -ycu can score your diet quickly. Its system has been simplified to avoid awkward decimals, as explained on the inside back cover. Suggestion for using it is to place a check $(V)$ beside each food you have eaten during one day. After you have done this, add the values of these foods on each double page. When all of the trages are in place you may then add up the figures for meat, vegetables, cereais, and so rorth, to obtain your total score. Finally, compare these with the recommended daily allowances on the last page. You may then discover whai fcods you resd to add to improve your diet.

The blank pages in the back may Dé used in plarning the pattern of meals in the total day's diet.

The number of foods which could be included in this booklet is necessarily limited. With some foods you will need to figure what is in the food. For example, vegetable stew:

Beef 1 portion

Carrots 1 portion
Potatoes 1 portion

Tomatoes $1 / 2$ portion

Gravy 1 portion 
UNITS (For conversion to grams or milligrams, see inside back cover.)

Food

Protein

Calcium

Iron

Vitamin A Vitamin C

Thia-

Ribo-

\section{PROTEINS}

Milk

Whole

1 cup (8 oz.)

$\begin{array}{rrr}165 & 85 & 28 \\ 330 & 170 & 56 \\ 495 & 255 & 84\end{array}$

$\begin{array}{rr}28 & 2 \\ 56 & 4 \\ 84 & 6 \\ 112 & 8\end{array}$

$\begin{array}{rrr}2 & 390 & 3 \\ 4 & 789 & 6 \\ 6 & 1,170 & 9 \\ 8 & 1,560 & 12\end{array}$

$\begin{array}{rl}3 & 10 \\ 6 & 20 \\ 9 & 30 \\ 12 & 40\end{array}$

\begin{tabular}{rrr}
10 & 40 & 3 \\
20 & 80 & 6 \\
30 & 120 & 9 \\
40 & 160 & 12 \\
\hline
\end{tabular}

Evap., vitamin D enriched

$1 / 2$ cup +

660

340

Skim, and nonfat buttermilk

$1 / 2$ cup water

Vitamin D enriched

1 cup $(8 \mathrm{oz}$.)

$\begin{array}{rr}175 & 88 \\ 85 & 85 \\ 165 & 85\end{array} \frac{30}{23}$

1 cup $(8 \mathrm{oz}$.)

\section{Meats and Fowl (For all fried meats and fowl, add 100 calories per seiving.)}

Bacon, crisp (See Fats)

(roasted or broiled)

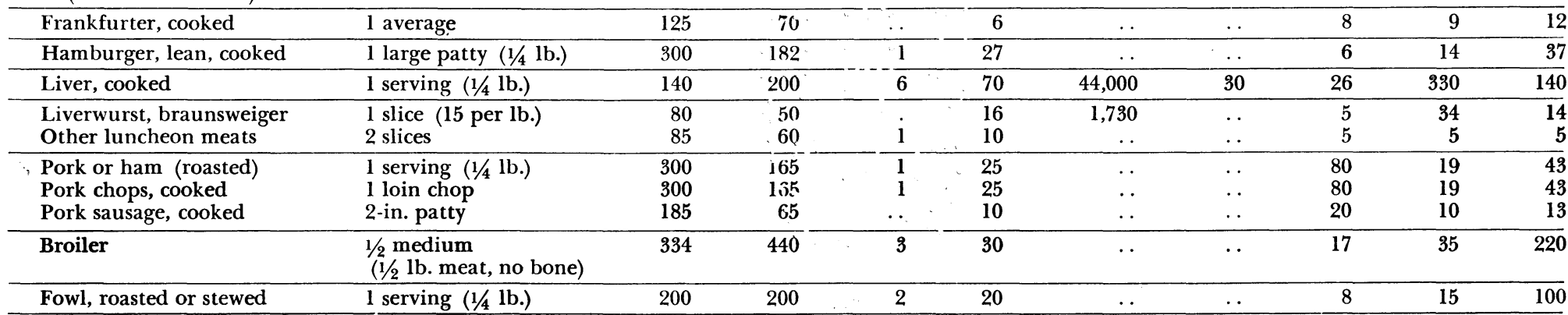

Fish and Shellfish (For all fried fish, add 100 calories per serving.)

Cod, haddock (cooked)

Halibut (cooked)

Lobster meat, cooked

Oysters, raw

stew

1 serving ( $1 / 4 \mathrm{lb}$.)

1 serving ( $1 / 4$ lb.)

$2 / 8$ cup

5-8 medium

4 oysters +

1 cup milk

\begin{tabular}{rr}
170 & 180 \\
\hline 200 & 210 \\
\hline 90 & 185 \\
\hline 85 & 100 \\
320 & 150
\end{tabular}

180

\begin{tabular}{l}
1 \\
\hline 2 \\
9 \\
35
\end{tabular}

\begin{tabular}{rrrr}
5 & $\ldots$ & $\ldots$ & 5 \\
\hline 8 & 500 & $\ldots$ & 6 \\
\hline 8 & $\ldots$ & $\ldots$ & 3 \\
\hline 56 & 320 & $\ldots$ & 15 \\
38 & 1,060 & 3 & 19 \\
\hline
\end{tabular}

\begin{tabular}{rr}
9 & 20 \\
\hline 6 & 90 \\
\hline 7 & 20 \\
\hline 20 & 12 \\
55 & 11 \\
\hline
\end{tabular}




\begin{tabular}{|c|c|c|c|c|c|c|c|c|c|c|}
\hline $\begin{array}{l}\text { Salmon, canned with bones } \\
\text { fresh, boneless }\end{array}$ & $\begin{array}{l}1 \text { serving }(2 / 3 \text { cup }) \\
1 \text { serving }(1 / 4 \mathrm{lb} .)\end{array}$ & $\begin{array}{l}140 \\
290\end{array}$ & $\begin{array}{l}205 \\
210\end{array}$ & $\begin{array}{r}19 \\
2\end{array}$ & $\begin{array}{l}8 \\
6\end{array}$ & $\begin{array}{r}70 \\
460\end{array}$ & $\begin{array}{l}. \\
\cdots\end{array}$ & $\begin{array}{r}3 \\
12\end{array}$ & $\begin{array}{l}18 \\
25\end{array}$ & $\begin{array}{l}80 \\
80\end{array}$ \\
\hline Sardines, canned & l sardine & 100 & 90 & 19 & 20 & 15 & $\ldots$ & 5 & 15 & 37 \\
\hline Shrimp, canned or cooked & $4-6$ medium & 65 & 135 & 6 & 16 & 30 & . & 1 & 2 & 10 \\
\hline Tuna, canned & $1 / 2$ cup & 200 & 290 & 1 & 14 & 80 & $\ldots$ & 5 & 12 & 130 \\
\hline \multicolumn{11}{|c|}{ Egas (For fried egg, add 100 calories.) } \\
\hline $\begin{array}{l}\text { boiled or poached } \\
\text { omelet or scrambled }\end{array}$ & $\begin{array}{l}1 \text { medium } \\
1 \text { egg }+2 \mathrm{~T} \text {. milk }\end{array}$ & $\begin{array}{r}80 \\
106\end{array}$ & $\begin{array}{l}60 \\
68\end{array}$ & $\begin{array}{l}3 \\
5\end{array}$ & $\begin{array}{l}15 \\
13\end{array}$ & $\begin{array}{l}550 \\
640\end{array}$ & $\begin{array}{l}\cdots \\
\cdots\end{array}$ & $\begin{array}{l}5 \\
5\end{array}$ & $\begin{array}{l}14 \\
17\end{array}$ & $\begin{array}{l}. \\
\cdots\end{array}$ \\
\hline $\begin{array}{l}\text { Cheese } \\
\text { American cheddar }\end{array}$ & 1-in. cube $(1 \mathrm{oz})$. & 115 & 71 & 20 & 3 & 400 & . & 1 & 12 & . \\
\hline Cheese spread & $1 \mathrm{oz}$. & 105 & 60 & 2 & $\ldots$ & $\ldots$ & $\ldots$ & $\ldots$ & $\ldots$ & . \\
\hline $\begin{array}{l}\text { Cottage cheese, dry } \\
\text { creamed }\end{array}$ & $\begin{array}{l}1 / 2 \text { cup } \\
1 / 2 \text { cup }\end{array}$ & $\begin{array}{r}95 \\
155 \\
\end{array}$ & $\begin{array}{l}195 \\
205\end{array}$ & $\begin{array}{l}10 \\
12 \\
\end{array}$ & $\begin{array}{l}3 \\
5 \\
\end{array}$ & $\begin{array}{r}20 \\
270 \\
\end{array}$ & $\begin{array}{l}\ldots \\
. . \\
\end{array}$ & $\begin{array}{c}2 \\
\ldots \\
\end{array}$ & $\begin{array}{l}30 \\
34 \\
\end{array}$ & 1 \\
\hline Processed cheese & $1 \mathrm{oz}$. & 90 & 50 & 17 & $\ldots$ & $\ldots$ & $\ldots$ & $\ldots$ & $\ldots$ & $\ldots$ \\
\hline \multicolumn{11}{|l|}{ Nuts } \\
\hline Almonds & $12-15$ & 90 & 28 & 4 & 7 & . & . & 4 & 10 & 7 \\
\hline Peanuts & $12-15$ & 85 & 40 & 1 & 3 & . & .. & 5 & 2 & 24 \\
\hline Pecan or walnut halves & $12-15$ & 100 & 20 & 1 & 4 & 7 & .. & 8 & 2 & 2 \\
\hline Peanut Butter & $1 \mathrm{~T}$. & 90 & 40 & 1 & 3 & $\ldots$ & . & 2 & 2 & 24 \\
\hline
\end{tabular}

\section{TOTAL MILK, MEAT, FISH, EGGS, CHEESE, NUTS}


F R U I T S (For sweetened canned or frozen fruit, add 50 calories per serving. For sweetened fresh fruit, add 20 calories per tsp. sugar used.)

\begin{tabular}{|c|c|c|c|c|c|c|c|c|c|c|}
\hline $\begin{array}{l}\text { Apple, raw } \\
\text { Applesauce, sweetened }\end{array}$ & $\begin{array}{l}1 \text { medium } \\
1 / 2 \text { cup }\end{array}$ & $\begin{array}{l}75 \\
90\end{array}$ & $\begin{array}{l}4 \\
3\end{array}$ & $\begin{array}{l}1 \\
1\end{array}$ & $\begin{array}{l}4 \\
5\end{array}$ & $\begin{array}{r}120 \\
40\end{array}$ & $\begin{array}{l}6 \\
1\end{array}$ & $\begin{array}{l}5 \\
3\end{array}$ & $\begin{array}{l}4 \\
2\end{array}$ & $\begin{array}{l}2 \\
1\end{array}$ \\
\hline Apricots & $2-3$ medium & 50 & 10 & 2 & 5 & 2,800 & 7 & 3 & 5 & $\overline{8}$ \\
\hline Banana & 1 medium & 130 & 18 & 1 & 9 & 650 & 15 & 6 & 8 & 10 \\
\hline Cantaloupe & $1 / 2\left(41 / 2^{\prime \prime}\right.$ diam.) & 30 & 9 & 3 & 6 & 5,000 & 50 & 8 & 6 & 7 \\
\hline $\begin{array}{l}\text { Cherries, sweet, raw, } \\
\text { sour, canned, unsweetened }\end{array}$ & $\begin{array}{l}15 \text { large } \\
1 / 2 \text { cup }\end{array}$ & $\begin{array}{l}60 \\
50\end{array}$ & $\begin{array}{r}11 \\
8\end{array}$ & $\begin{array}{l}2 \\
1\end{array}$ & $\begin{array}{l}4 \\
3\end{array}$ & $\begin{array}{l}620 \\
720\end{array}$ & $\begin{array}{l}8 \\
6\end{array}$ & $\begin{array}{l}5 \\
3\end{array}$ & $\begin{array}{l}6 \\
2\end{array}$ & $\begin{array}{l}4 \\
6\end{array}$ \\
\hline $\begin{array}{l}\text { Citrus - orange, grapefruit, } \\
\text { lemon }\end{array}$ & $\begin{array}{l}1 \text { orange or lemon or } \\
1 / 2 \text { grapefruit or } \\
1 / 2 \text { cup juice }\end{array}$ & 50 & 9 & 3 & 4 & 180 & 50 & 8 & 3 & 3 \\
\hline Dried dates, figs, raisins & $1 / 4$ cup & 80 & 5 & 3 & 9 & 15 & $\ldots$ & 4 & 3 & 6 \\
\hline Fruit cocktail, canned & $1 / 2$ cup & 90 & 5 & I & 5 & 205 & 3 & 2 & 2 & $\overline{5}$ \\
\hline Grapes & 1 bunch (22 to 24$)$ & 70 & 14 & 2 & 6 & 80 & 4 & 6 & 4 & 2 \\
\hline Peach, raw & 1 medium & 50 & 5 & 1 & 6 & 880 & 8 & 2 & 5 & 9 \\
\hline Pear, raw & 1 medium & 60 & 1 & 2 & 3 & 20 & 4 & 2 & 4 & $\overline{1}$ \\
\hline $\begin{array}{l}\text { Pineapple, canned, sweetened } \\
\text { fresh, unsweetened }\end{array}$ & $\begin{array}{l}1 / 2 \text { cup } \\
1 / 2 \text { cup }\end{array}$ & $\begin{array}{r}100 \\
50\end{array}$ & $\begin{array}{l}5 \\
4\end{array}$ & $\begin{array}{l}4 \\
2\end{array}$ & $\begin{array}{l}8 \\
3\end{array}$ & $\begin{array}{l}100 \\
130\end{array}$ & $\begin{array}{l}12 \\
24\end{array}$ & $\begin{array}{r}10 \\
8\end{array}$ & $\begin{array}{l}2 \\
2\end{array}$ & $\begin{array}{l}2 \\
2\end{array}$ \\
\hline Plums, raw & 2 medium & 50 & 7 & 2 & 5 & 350 & 5 & 6 & 4 & $\overline{5}$ \\
\hline Rhubarb, cooked, sweetened & $1 / 2$ cup & 140 & 3 & $\ldots$ & 2 & 16 & 2 & . & . & $\bar{\cdots}$ \\
\hline Strawberries & 10 large & 40 & 8 & 3 & 8 & 60 & 60 & 3 & 7 & 3 \\
\hline Watermelon & $6^{\prime \prime}$ diam. $\times 11 / 2 "$ & 170 & 3 & 4 & 12 & 3,540 & 35 & 30 & 30 & 12 \\
\hline
\end{tabular}




\section{DES SERT S}

Cakes

Angel

Chocolate, white icing

Fruit

$1 / 10$ th of $10^{\prime \prime}$ cake 1/12th of 9 " cake

Plain, white icing

Cookies, plain, sugar Chocolate brownie 3 " $\times 3$ " $\times 1 / 2$ " Fruit nut (rich)

3 " $\times 2$ " $\times 13 / 4 "$

Custard, baked

2 (3" diam.)

$2^{\prime \prime} \times 2$ " $\times 3 / 4 "$

2 (3" diam.)

Doughnuts, cake type raised

$1 / 2$ cup

Gelatin dessert, plain

1 medium

1 medium

\begin{tabular}{l}
150 \\
360 \\
140 \\
300 \\
130 \\
140 \\
230 \\
\hline
\end{tabular}

$\begin{array}{llr}35 & \ldots & \\ 30 & 2 & \\ 20 & 4 & 10 \\ 38 & 8 & \end{array}$

11.

$20 \quad 1 \quad 450$

$\begin{array}{rrrr}20 & 1 & 4 & 50 \\ 18 & 1 & 5 & 230 \\ 15 & 1 & 5\end{array}$

$\begin{array}{rrr}15 & 1 & \\ 90 & 16 & 10\end{array}$

$140 \quad 20$

$20 \quad 1 \quad 6 \quad 60$

120

20

$\begin{array}{lll}20 & 2 & 4\end{array}$

Ice cream, vanilla

$1 / 2$ cup

70

16

$150 \quad 28$

$\begin{array}{lll}28 & 9 & 1\end{array}$

370

1

3

13

Pies

Blueberry $\quad 1 / 6$ of 9" pie

Cream filling, with meringue $1 / 6$ of 9 " pie

Fruit (apple, peach, cherry) $1 / 6$ of 9" pie

Pumpkin

Pudding, cream style

$1 / 6$ of 9" pie

$1 / 2$ cup

370
350
380
330
150

$\begin{array}{rrrr}40 & 1 & 7 & 17 \\ 75 & 5 & 8 & 300 \\ 40 & 2 & 6 & 500 \\ 65 & 10 & 20 & 2,300 \\ 45 & 15 & 1 & 200\end{array}$

170

300

500

2,300

200

$\begin{array}{rrrr}5 & 3 & 3 & 4 \\ \ldots & 7 & 24 & 2 \\ 2 & 5 & 4 & 6 \\ \ldots & 6 & 16 & 5 \\ \ldots & 4 & 20 & 1\end{array}$

- TOTAL FRUITS and DESSERTS 


\section{VEGETABLES}

Asparagus, cooked canned green

$2 / 3$ cup

6 medium stalks

\begin{tabular}{ll}
20 & 24 \\
20 & 24 \\
\hline
\end{tabular}

Beans, green, cooked canned

$1 / 2$ cup

lima, green, frozen, cooked

$1 / 2$ cup

$1 / 2$ cup

$\begin{array}{rr}15 & 9 \\ 20 & 12\end{array}$

Beets, cooked
Broccoli, cooked

$1 / 2$ cup

$110 \quad 65$

24
24
9
12
65

Brussels sprouts, cooked

$2 / 3$ cup

$35 \quad 8$

Cabbage, raw cooked

$30 \quad 3$

82

Sauerkraut $1 / 2$ cup

$30 \quad 30$

Carrots, raw

$\begin{array}{ll}1 / 2 & \text { cup } \\ 1 / 2 & \text { cup } \\ 2 / 3 & \text { cup }\end{array}$

157

13

210

$10 \quad 1,040 \quad 23$

23
18

$\begin{array}{lll}13 & 17 & 12 \\ 6 & 8\end{array}$

$2 \quad 19 \quad 800$

315

500

220

5

$\begin{array}{rrr}4 & 315 & 5 \\ 15 & 500 & 5 \\ 19 & 220 & 20\end{array}$

20

10

$\begin{array}{ll}\begin{array}{l}\text { Carrots, raw } \\ \text { cooked }\end{array} & 1 \text { large } \\ 2 / 3 \text { cup }\end{array}$

\begin{tabular}{ll}
\hline Cauliflower, cooked & $1 / 2$ cup \\
\hline $\begin{array}{c}\text { Corn, cooked } \\
\text { canned }\end{array}$ & 1 ear $5^{\prime \prime}$ long \\
\hline
\end{tabular}

\section{Dried navy beans,} peas, cooked

$1 / 2 \operatorname{cup}$

115

$25 \quad 12$

7

$\begin{array}{lllll}40 & 12 & 2 & 8 & 12,000\end{array}$

20

$\begin{array}{ll}15 & 15 \\ 85 & 27 \\ 90 & 39\end{array}$

2

Leafy greens

Beet tops, spinach, cooked

$2 / 3$ cup

30

25

25

10,000

33

33

8




\begin{tabular}{|c|c|c|c|c|c|c|c|c|c|c|}
\hline $\begin{array}{l}\text { Lettuce } \\
\text { Celery } \\
\text { Cucumber }\end{array}$ & $\begin{array}{l}1 / 8 \text { head } \\
2 \text { pieces } \\
8 \text { slices }\end{array}$ & 10 & 5 & 2 & 2 & 100 & 4 & 2 & 3 & 2 \\
\hline Onions, egg plant, cooked & $1 / 2$ cup & 40 & 10 & 3 & 6 & 80 & 8 & 3 & 4 & 3 \\
\hline $\begin{array}{l}\text { Peas, green, cooked } \\
\text { canned }\end{array}$ & $\begin{array}{l}1 / 2 \text { cup } \\
1 / 2 \text { cup }\end{array}$ & $\begin{array}{l}60 \\
70\end{array}$ & $\begin{array}{l}38 \\
36\end{array}$ & $\begin{array}{l}2 \\
3\end{array}$ & $\begin{array}{l}15 \\
17\end{array}$ & $\begin{array}{l}600 \\
550\end{array}$ & $\begin{array}{r}12 \\
8\end{array}$ & $\begin{array}{l}20 \\
10\end{array}$ & $\begin{array}{r}11 \\
5\end{array}$ & $\begin{array}{r}20 \\
8\end{array}$ \\
\hline \multicolumn{11}{|l|}{ Potatoes } \\
\hline $\begin{array}{l}\text { Pickles, sweet cucumber } \\
\text { Dill }\end{array}$ & $\begin{array}{l}1 \text { medium } \\
1 \text { large }\end{array}$ & $\begin{array}{l}10 \\
15\end{array}$ & $\begin{array}{l}1 \\
9\end{array}$ & $\begin{array}{l}1 \\
4\end{array}$ & $\begin{array}{l}1 \\
2\end{array}$ & $\begin{array}{r}10 \\
420\end{array}$ & $\ddot{8}$ & $\begin{array}{l}\cdots \\
\cdots\end{array}$ & $\ddot{8}$ & $\begin{array}{l}\cdots \\
\cdots\end{array}$ \\
\hline Rutabagas, cooked & $1 / 2$ cup & 25 & 6 & 4 & 3 & 270 & 16 & 4 & 6 & 5 \\
\hline $\begin{array}{l}\text { Tomatoes, canned } \\
\text { juice, canned } \\
\text { raw }\end{array}$ & $\begin{array}{l}1 / 2 \text { cup } \\
1 / 2 \text { cup } \\
1 \text { medium }\end{array}$ & $\begin{array}{l}25 \\
20 \\
30\end{array}$ & $\begin{array}{l}12 \\
10 \\
15\end{array}$ & $\begin{array}{l}1 \\
1 \\
2\end{array}$ & $\begin{array}{l}7 \\
4 \\
9\end{array}$ & $\begin{array}{l}1,250 \\
1,050 \\
1,650\end{array}$ & $\begin{array}{l}20 \\
16 \\
35\end{array}$ & $\begin{array}{l}7 \\
5 \\
9\end{array}$ & $\begin{array}{l}4 \\
3 \\
6\end{array}$ & $\begin{array}{l}8 \\
8 \\
8\end{array}$ \\
\hline Turnips, cooked & $2 / 3$ cup & 25 & 8 & 4 & 5 & .. & 18 & 4 & 6 & 4 \\
\hline \multicolumn{2}{|c|}{ For buttered vegetables, add per serving: } & 50 & . & . & .. & 200 & $\ldots$ & .. & $\ldots$ & $\cdots$ \\
\hline
\end{tabular}




\section{BREADS A D CEREAL FOODS}

Enriched white or whole grain

Bread

1 slice $\}$

$1 \quad 65$

130

20

40

Muffin, plain

1 av.

1 med.

$1\left(4^{\prime \prime} \times 4^{\prime \prime}\right)$

$1\left(5^{\prime \prime} \times 5^{\prime \prime} \times 1 / 2^{\prime \prime}\right)$

$3 \quad 195$

120

60

32

Roll, plain, pan

80

60

Waffle

$1 / 2$ cup

$1 / 2$ cup

1 cup

1 cup

1 cup

216

21

18

18
70

2
4
6
3
2
4
14

4

8

8

7

5

4

14

$\cdots$
$\cdots$
190
75
50
270

.

6

12

4

8

12

10

6

6

20

10

15

6

5

3

10

Cereal, cooked

Oatmeal or rolled oats

Wheat

Cereal, ready-to-eat, flake

sugar-coated

\section{1 cracker}

Crackers, graham

1 cracker

$\begin{array}{rr}75 & 25 \\ 70 & 28 \\ 115 & 27 \\ 49 & 12 \\ 110 & 12\end{array}$

\begin{tabular}{l}
25 \\
28 \\
27 \\
12 \\
12 \\
\hline 5 \\
7
\end{tabular}

1
1
1
3
3

\begin{tabular}{l}
8 \\
7 \\
9 \\
4 \\
5 \\
\hline 1 \\
1
\end{tabular}

Egg noodles, enriched, cooked 1 cup

Macaroni, rice, spaghetti

unenriched, cooked

1 cup

107

35

8

209

62

2

7

50

1 strip, crisp

35

18

1 teaspoon

.

.

2

..

165

$\begin{array}{llr}\ldots & \ldots & 11 \\ \ldots & \ldots & 9 \\ \ldots & \ldots & 12 \\ \ldots & \ldots & 7 \\ \ldots & \ldots & 13 \\ \ldots & \ldots & 2 \\ \ldots & \ldots & 1\end{array}$

\begin{tabular}{rr}
3 & 2 \\
5 & 7 \\
4 & 14 \\
2 & 11 \\
2 & 10 \\
\hline 1 & 1 \\
\hline 1 & $\ldots$ \\
\hline 10 & 17 \\
\hline 2 & 7 \\
\hline
\end{tabular}

\begin{tabular}{ccccc}
60 & $\ldots$ & 22 & 10 & 17 \\
$\ldots$ & $\ldots$ & 3 & 2 & 7 \\
\hline
\end{tabular}

Bacon

Butter or fortified margarine 


\begin{tabular}{|c|c|c|c|c|c|c|c|c|c|c|}
\hline $\begin{array}{c}\text { Cream, light (coffee) } \\
\text { heavy (whipping) }\end{array}$ & $\begin{array}{l}2 \text { T. ( } 1 / 8 \text { cup) } \\
2 \text { T. ( } 1 / 8 \text { cup) }\end{array}$ & $\begin{array}{r}60 \\
100 \\
\end{array}$ & $\begin{array}{l}8 \\
6 \\
\end{array}$ & $\begin{array}{l}2 \\
2 \\
\end{array}$ & $\begin{array}{l}\cdots \\
\cdots \\
\end{array}$ & $\begin{array}{l}250 \\
440 \\
\end{array}$ & $\begin{array}{l}\cdots \\
\cdots \\
\end{array}$ & $\begin{array}{l}\cdots \\
\cdots \\
\end{array}$ & $\begin{array}{l}2 \\
4 \\
\end{array}$ & $\begin{array}{l}\cdots \\
\cdots\end{array}$ \\
\hline \multicolumn{11}{|c|}{ Shortenings (Add these values to any fried food not given a listing as fried.) } \\
\hline Beef suet, rendered & $1 \mathrm{~T}$. & 120 & 2 & . & . & 70 & .. & . & . & $\ldots$ \\
\hline Lard & $1 \mathrm{~T}$. & 125 & . & . & . & . & .. & . & .. & . \\
\hline \multicolumn{11}{|l|}{ Foods with high fat content } \\
\hline Gravy (with milk) & $1 / 4$ cup & 100 & 25 & 7 & 2 & 225 & . & 4 & 11 & 2 \\
\hline Mayonnaise & $1 \mathrm{~T}$ & 90 & 2 & . & 1 & 35 & . & . & .. & .. \\
\hline Salad dressing (French) & $1 \mathrm{~T}$. & 60 & 1 & . & .. & . & . & . & . & . \\
\hline White sauce (medium) & $2 \mathrm{~T}$. & 50 & 13 & 4 & 1 & 165 & . & 1 & 5 & 1 \\
\hline \multicolumn{11}{|l|}{ SWEET S } \\
\hline Chocolate-coated candy bar & $2 \mathrm{oz}$. & 270 & 52 & 5 & 14 & . & . & 3 & 9 & 3 \\
\hline Honey, strained & $1 \mathrm{~T}$. & 60 & 1 & . & 2 & . & . & .. & 1 & .. \\
\hline Jam, jelly, syrup & $1 \mathrm{~T}$. & 55 & . & . & . & . & . & .. & . & .. \\
\hline Molasses, medium dark & $1 \mathrm{~T}$. & 45 & $\ldots$ & 6 & 12 & . & $\ldots$ & .. & $\ldots$ & .. \\
\hline Sugar, granulated & $1 \mathrm{~T}$. & 50 & . & .. & . & . & .. & .. & . & .. \\
\hline brown, dark & $1 \mathrm{~T}$. & 50 & $\cdots$ & 1 & 4 & . & . & $\cdots$ & $\cdots$ & ․ \\
\hline \multicolumn{11}{|l|}{ BEVERAG ES } \\
\hline Chocolate malted milk shake & 1 serving (8 oz. milk) & 500 & 130 & 42 & 13 & 900 & 4 & 19 & 65 & 5 \\
\hline Cocoa or chocolate & 1 cup $(6$ oz. milk) & 180 & 70 & 22 & 9 & 300 & 2 & 8 & 33 & 3 \\
\hline Grape juice, sweetened & 3 oz. & 70 & 4 & 1 & 3 & . & . & 4 & 5 & 2 \\
\hline Soft drinks & 1 bottle $(6 \mathrm{oz})$. & 80 & .. & . & .. & .. & . & .. & .. & .. \\
\hline
\end{tabular}

\section{totAl Cereals, fats, SWEets, BeVERAGeS}




\section{RECOMMENDED DAILY ALLOWANCES EXPRESSED IN UNITS}

Adapted with practical modifications from the Recommended Dietary Allowances of the National Research Council, 1953. Refer to inside back cover for explanation of units.

\begin{tabular}{|c|c|c|c|c|c|c|c|c|c|c|c|c|c|}
\hline \multirow[b]{2}{*}{ - } & \multirow[b]{2}{*}{$\begin{array}{c}\text { Age } \\
\text { (years) }\end{array}$} & \multirow[b]{2}{*}{$\begin{array}{l}\text { Weight } \\
\text { (lbs.) }\end{array}$} & \multirow[b]{2}{*}{$\begin{array}{l}\text { Height } \\
\text { (in.) }\end{array}$} & \multirow[b]{2}{*}{$\underset{\text { Units }}{\text { Vitamin D }}$} & \multirow[b]{2}{*}{ Calories } & \multicolumn{8}{|c|}{ UNITS } \\
\hline & & & & & & Protein & Calcium & Iron & Vitamin A & Vitamin C & $\begin{array}{l}\text { Thia- } \\
\text { mine }\end{array}$ & $\begin{array}{l}\text { Ribo- } \\
\text { flaỵin }\end{array}$ & Niacin \\
\hline \multicolumn{14}{|c|}{ CHILDREN } \\
\hline & $1-3$ & 27 & 34 & 800 & 1,200 & 400 & 100 & 70 & 2,000 & 35 & 60 & 100 & 60 \\
\hline & 4-6 & 40 & 43 & 800 & 1,600 & 500 & 100 & 80 & 2,500 & 50 & 80 & 120 & 80 \\
\hline & $7-9$ & 59 & 51 & 800 & 2,000 & 600 & 100 & 100 & 3,500 & 60 & 100 & 150 & 100 \\
\hline \multicolumn{14}{|l|}{ BOYS } \\
\hline & $10-12$ & 78 & 57 & 800 & 2,500 & 700 & 120 & 120 & 4,500 & 75 & 130 & 180 & 130 \\
\hline & $13-15$ & 108 & 64 & 800 & 3,200 & 850 & 140 & 150 & 5,000 & 90 & 160 & 210 & 160 \\
\hline & $16-20$ & 139 & 69 & 800 & 3,800 & 1,000 & 140 & 150 & 5,000 & 100 & 190 & 250 & 190 \\
\hline \multicolumn{14}{|l|}{ GIRLS } \\
\hline & $10-12$ & 79 & 57 & 800 & 2,300 & 700 & 120 & 120 & 4,500 & 75 & 120 & 180 & 120 \\
\hline & $13-15$ & 108 & 63 & 800 & 2,500 & 800 & 130 & 150 & 5,000 & 80 & 130 & 200 & 130 \\
\hline & $16-20$ & 120 & 64 & 800 & 2,400 & 750 & 130 & 150 & 5,000 & 80 & 120 & 190 & 120 \\
\hline \multicolumn{14}{|l|}{ MEN } \\
\hline & 25 & 143 & 67 & & 3,200 & 650 & 80 & 120 & 5,000 & 75 & 160 & 160 & 160 \\
\hline & 45 & 143 & 67 & & 2,900 & 650 & 80 & 120 & 5,000 & 75 & 150 & 160 & 150 \\
\hline & 65 & 143 & 67 & & 2,600 & 650 & 80 & 120 & 5,000 & 75 & 130 & 160 & 130 \\
\hline \multicolumn{14}{|l|}{ WOMEN } \\
\hline & 25 & 121 & 62 & & 2,300 & 550 & 80 & 120 & 5,000 & 70 & 120 & 140 & 120 \\
\hline & 45 & 121 & 62 & & 2,100 & 550 & 80 & 120 & 5,000 & 70 & 110 & 140 & 110 \\
\hline & 65 & 121 & 62 & & 1,800 & 550 & 80 & 120 & 5,000 & 70 & 100 & 140 & 100 \\
\hline Pregnant & (3rd trimester) & & & 800 & Add $\quad 400$ & 800 & 150 & 150 & 6,000 & 100 & 150 & 200 & 150 \\
\hline \multirow[t]{2}{*}{ Lactating } & & & & 800 & Add 1,000 & 1,000 & 200 & 150 & 8,000 & 150 & 150 & 250 & 150 \\
\hline & & & & & Calories & Protein & Calcium & Iron & Vitamin A & Vitamin C & $\begin{array}{l}\text { Thia- } \\
\text { mine }\end{array}$ & $\begin{array}{l}\text { Ribo- } \\
\text { flavin }\end{array}$ & Niacin \\
\hline
\end{tabular}

